\title{
Aprender finanzas conductuales experimentando
}

\author{
Irene Comeig Ramírez ${ }^{\mathrm{a}}$ y Pau Sendra Pons ${ }^{\mathrm{b}}$
}

${ }^{a}$ Departamento de Finanzas Empresariales y ERI-CES, Facultat d'Economia (Universitat de València), Avda. de los Naranjos, s/n, 46071, València (irene.comeig@uv.es) b Departamento de Finanzas Empresariales, Facultat d’Economia (Universitat de València), Avda. de los Naranjos, s/n, 46071, València (pau.sendra-pons@uv.es)

\section{\$EWWDW}

, QDQQHMHUFKDQJ LQJ

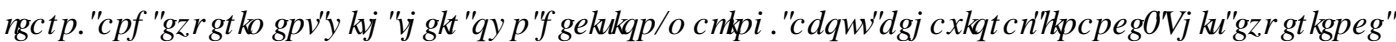

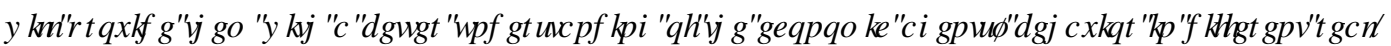

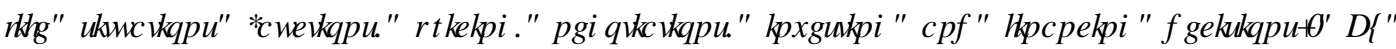

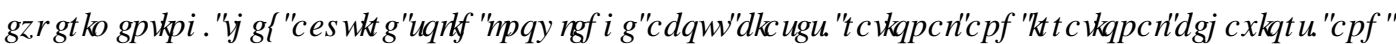

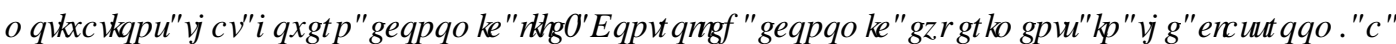

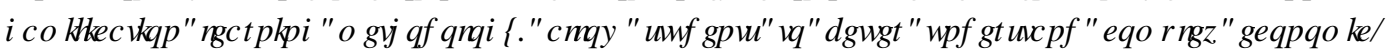

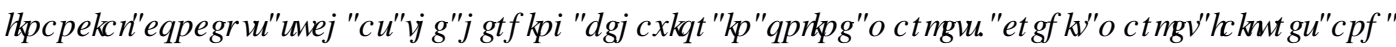

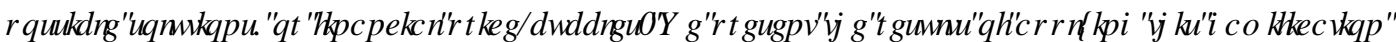

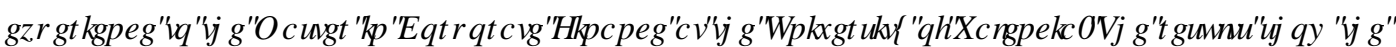

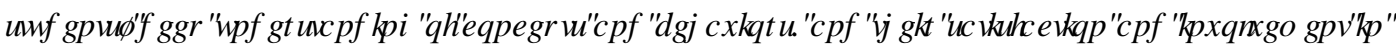

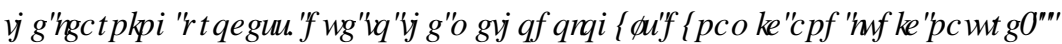

\section{. H Z RLGVIEH KDVIRLOOIQDQFHIH SHUP HQWDOHRQRP IFVUJDP IIFDURQIKUJKHUHGXFDURQI}

[

\section{HXP HQ}

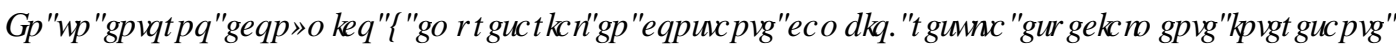

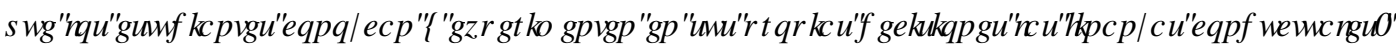

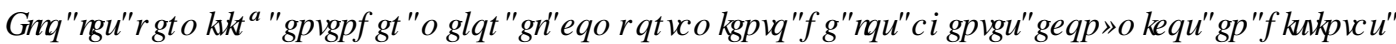

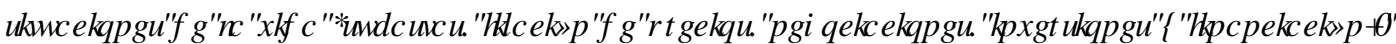

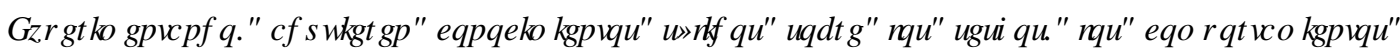

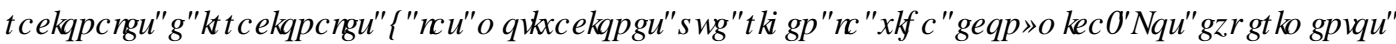

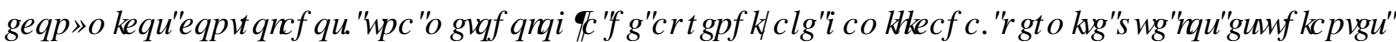
DSUHQCDQ MUJDQCR I I FRP SUHQDDQ P HRU FRQFHS URV HFRQ P IFR IIQDQFHHRV FRP SCHNV FRP R! HD

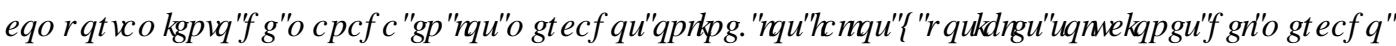

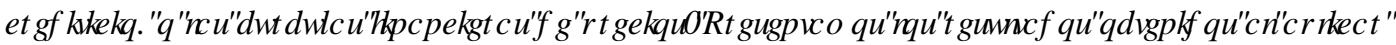

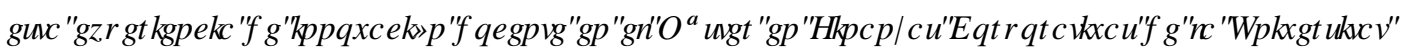
GH 9 DQQFWD ' IFKRV UHXQDORV FRURERWQ QD SLRIXQDD FRP SUHQYY TXH FRQMJXIHRQ QRV

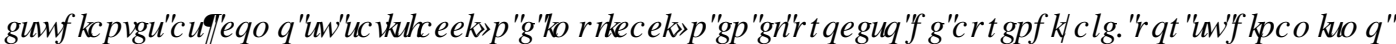
I IFDU FHUOGFRT

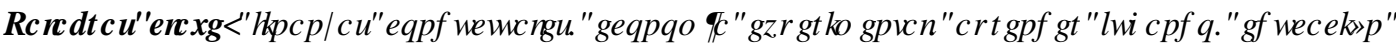
XSHURU 


\section{Introducción}

El estudio de cómo los individuos toman decisiones en su vida diaria ha dado lugar al nacimiento de nuevas áreas de investigación como el derecho conductual, la economía del comportamiento o la neuroeconomía, basadas en experimentos de laboratorio y de campo (Holt, 2019, p. XI). Cada vez más, el estudio de aspectos económicos incorpora consideraciones sociales y psicológicas, en línea con las aportaciones de los Premios Nobel Vernon L. Smith (2002), Daniel Kahneman (2002), Robert H. Thaler (2017), Michael Kremer (2019), Abhijit Banerjee (2019) y Esther Duflo (2019). Vernon L. Smith, Doctor en Economía por la Universidad de Harvard, fue galardonado con el Premio Nobel en Ciencias Económicas "por haber establecido experimentos de laboratorio como una herramienta para el análisis económico empírico, especialmente en el estudio de mecanismos alternativos de mercado". El propio Smith identifica numerosas razones por las que los economistas deberían llevar a cabo experimentos, entre las que destacan su utilidad a la hora de analizar una teoría o discriminar entre teorías, comparando las predicciones teóricas con las observaciones experimentales; explorar las causas del fracaso de una teoría, cuando las observaciones no avalan las hipótesis iniciales; y establecer regularidades empíricas como base para una nueva teoría (Smith, 1994).

La principal característica de los experimentos económicos es que permiten inferir causalidad. Mediante un análisis estadístico podemos establecer una correlación entre variables, sin embargo, para definir una relación causal es necesario un enfoque experimental. Asimismo, los experimentos son replicables lo que permite hallar patrones culturales, geográficos o de cualquier otra índole variando los sujetos a los que se dirige el experimento. Además, se desarrollan en ambientes controlados, donde las diferencias entre los distintos tratamientos (secciones del experimento) se establecen para evaluar el comportamiento de los sujetos ante cambios en las variables examinadas.

Dada la relevancia de las finanzas conductuales como disciplina económica, y la efectividad de las técnicas de aprendizaje basadas en el juego y la experimentación en la educación superior para la adquisición de conocimientos sólidos, hemos desarrollado un proyecto de innovación educativa que aúna tres técnicas relevantes. Estas tres metodologías que se combinan son: aprender experimentando, cooperar con el equipo, y aprender jugando en entornos digitales (JDP IIFDFlyQ). Esta innovación educativa se ha llevado a cabo con estudiantes del Máster Universitario en Finanzas Corporativas (MFC) de la Universitat de València, durante el curso 2019/20.

El aprendizaje cooperativo, consistente en el trabajo conjunto de estudiantes para conseguir objetivos comunes o completar tareas grupales, ha sido ampliamente avalado como una práctica pedagógica que fomenta la socialización y el aprendizaje, independientemente del nivel educativo y el área de estudio (Gillies, 2016). Según Cohn (1999), el aprendizaje cooperativo promueve la generación de nuevas ideas y soluciones y una mayor motivación por aprender, en comparación con el aprendizaje competitivo e individualista. En el MFC, la cooperación entre los estudiantes resultó crucial tanto para organizar y desarrollar los experimentos en clase, como para exponer los resultados de la experiencia, y para elaborar un informe.

Las otras metodologías aplicadas en esta experiencia de innovación docente, aprender experimentando (ADQTQJEEITRQ) y la JDP IIFDFIy se enmarcan dentro del aprendizaje activo, que incluye técnicas dirigidas a involucrar a los estudiantes en el proceso de aprendizaje dándoles un papel relevante en su propia formación (Hackathorn HWDO, 2011). La JDP IIFDFFy consiste en el uso de elementos y mecanismos propios del juego (a menudo $R Q Q Q Q H$ ) con el objetivo de aumentar la motivación de los estudiantes aprovechando e influyendo en los deseos naturales de los individuos por competir y ser reconocidos por sus resultados. Así pues, este tipo de aprendizaje permite consolidar mejor los conocimientos y recordarlos por más tiempo (Al-Azawi HWDO, 2016). Por su parte, los experimentos 
económicos controlados, con sus respectivas recompensas asociadas al desempeño de los sujetos, además de incorporar rigor científico, permiten introducir el componente lúdico en el aprendizaje de aspectos económico-financieros. El principal entorno digital que hemos utilizado para la realización de los experimentos económicos ha sido 9HFQDEEII ([SHUPHQWDO (FRQRPIFV] / DERLWRUL (veconlab.econ.virginia.edu), el cual, de una forma sencilla y ordenada, facilita la recopilación de las respuestas de los distintos participantes, el análisis de los resultados y la presentación de las conclusiones. Este tipo de plataformas RQDQH propician un entorno de aprendizaje atrayente e intuitivo para los alumnos. Tal y como muestran los resultados que se presentan posteriormente, los estudiantes demostraron un alto grado de iniciativa y liderazgo, y mejoraron significativamente sus conocimientos, para lo que contaron con las guías y pautas dadas por el profesorado.

A continuación se presenta el detalle de los objetivos de esta experiencia de innovación docente, del desarrollo de la innovación, y de los resultados alcanzados. Finalmente, en el cuarto apartado, se exponen las conclusiones alcanzadas.

\title{
1. Objetivos
}

Esta experiencia de innovación educativa ha tenido como objetivo principal enseñar de forma sólida y duradera las finanzas conductuales a los alumnos del Máster Universitario en Finanzas Corporativas (MFC) de la Universitat de València, favoreciendo la interiorización de los conocimientos. Al utilizar una metodología lúdica a través de experimentos económicos controlados en clase, se reduce la carga teórica de la asignatura sin renunciar a los estándares científicos, ya que los alumnos aprenden jugando y experimentando. El objetivo es consolidar firmemente conceptos económico-financieros complejos dado que los alumnos descubren por ellos mismos sus implicaciones lo que, a su vez, les ayuda a entender y recordar por más tiempo los conocimientos adquiridos (Ramírez y Grau, 2016; Comeig y JaramilloGutiérrez, 2011). Además, contribuye a ofrecer formación de postgrado alineada con las aportaciones de recientes premios Nobel como Daniel Kahneman (2002) o Richard H. Thaler (2017) mediante el uso de técnicas vanguardistas e innovadoras.

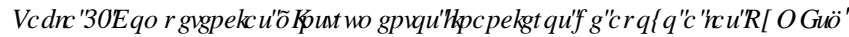

\begin{abstract}
Que los/las estudiantes sepan aplicar los conocimientos adquiridos y su capacidad de resolución de problemas en entornos nuevos o poco conocidos dentro de contextos más amplios (o multidisciplinares) relacionados con su área de estudio.
\end{abstract}

Capacidad para preparar, redactar y exponer en público informes y proyectos sobre política económica y economía pública de manera clara y coherente, defenderlos con rigor y tolerancia y responder satisfactoriamente a críticas sobre los mismos.

Que los/las estudiantes posean las habilidades de aprendizaje que les permitan continuar estudiando de un modo que habrá de ser en gran medida autodirigido o autónomo.

Trabajar en equipo con eficacia y eficiencia tanto en el área financiera como en las otras áreas funcionales de la empresa.

Fuente: Guía docente de la asignatura "Instrumentos financieros de apoyo a las PYMEs".

Por otro lado, esta metodología docente, eminentemente práctica y participativa, permite desarrollar con profundidad las competencias ya contempladas en la guía docente de la asignatura (ver Tabla 1). Estas competencias no se pueden trabajar adecuadamente con los sistemas tradicionales de aprendizaje basados en la clase magistral. De este modo, se persigue que los estudiantes puedan aplicar los conocimientos adquiridos a través de los experimentos a situaciones diarias de carácter económico-financiero como subastas o sistemas de fijación de precios y negociaciones. También se pretende desarrollar la capacidad de gestionar proyectos y presentar sus resultados de forma efectiva así como de ser proactivos para la

(c)) EY-NC-ND 2021, Universitat Politècnica de València

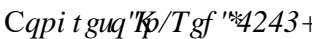


consecución de los objetivos fijados tanto individual como grupalmente. El trabajo en equipo juega un papel fundamental en esta innovación docente ya que el éxito del informe final requiere de la adecuada interacción entre los estudiantes constituidos en grupos de trabajo.

\section{Implementación de la innovación}

\subsection{Formulación de la tarea

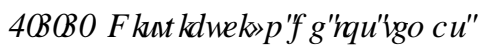

Los estudiantes fueron distribuidos aleatoriamente en grupos de trabajo formados por cuatro personas. A cada grupo se le asignó un tema relacionado con la asignatura que podía ser tratado desde un punto de vista experimental, es decir, realizando experimentos económicos en clase para interiorizar mejor la esencia de los conceptos tratados o la dinámica de los mecanismos financieros expuestos. La Tabla 2 resume la distribución de los grupos y la asignación de contenidos.

7DE(D) $\square 7$ H DVGHCRVWDEDKV

\begin{tabular}{cl}
\hline Grupo 1 & $\begin{array}{l}\text { El micromecenazgo, la financiación de las PYMEs y el } \\
\text { comportamiento de manada. }\end{array}$ \\
\hline Grupo 2 & $\begin{array}{l}\text { Las Sociedades de Garantía Recíproca (SGR) y la información } \\
\text { asimétrica. }\end{array}$ \\
\hline Grupo 3 & El Mercado Alternativo Bursátil (MAB) y las burbujas de precios. \\
\hline Grupo 4 & La fijación de precios mediante subastas. \\
\hline Grupo 5 & La teoría de agencia. Contratos Principal-Agente. \\
\hline Grupo 6 & Préstamos bancarios, interés y garantía. \\
\hline Grupo 7 & La negociación de precios y cantidades. \\
\hline Grupo 8 & La teoría prospectiva y la aversión a las pérdidas. \\
\hline
\end{tabular}

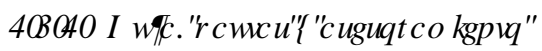

En el proceso de adquisición de conocimientos, la profesora Irene Comeig guió a los estudiantes a través de sesiones individuales con cada grupo. En el primer encuentro, se trataron aspectos generales sobre la temática asignada, instruyendo a los estudiantes sobre fuentes relevantes de las que obtener información. El papel del docente tuvo dos objetivos principales: orientar a los estudiantes sobre los aspectos formales que debían seguir tanto en la preparación de la exposición oral como en la redacción del informe, y proveerles de recursos bibliográficos con los que empezar afrontar el reto. Adicionalmente, la docente introdujo a los estudiantes en el mundo de la economía experimental, explicándoles el funcionamiento del experimento que debían replicar en clase así como asesorándoles en la interpretación de los resultados.

\subsection{Exposición del trabajo}

La exposición del trabajo de cada grupo, de 50 minutos de duración, tuvo dos partes diferenciadas: en primer lugar, la ejecución del experimento, conducida por los integrantes del grupo y en la que el resto de los estudiantes participó como sujetos experimentales; en segundo lugar, la presentación de los resultados del experimento obtenidos a partir de las decisiones de los estudiantes de la clase y, tras ello, una vez todos habían observado los resultados reales y estaban ansiosos por conocer el por qué, la explicación de los conceptos teóricos más importantes relacionados con el área de estudio. 


\section{૧प⿴囗 ( NAFXFYQGHOHSHUP HQMR]}

La mayoría de los experimentos se realizaron a través de la web 9HRQDEE(veconlab.econ.virginia.edu), creada por el reputado profesor experimentalista Charles A. Holt, de la Universidad de Virginia (EEUU). Esta plataforma permite realizar experimentos relacionados con aspectos económico-financieros con gran flexibilidad, ya que admite cambiar parámetros para adaptarlos a diferentes situaciones. Este entorno digital guía a los sujetos a través del experimento de forma muy intuitiva, como un juego RQQQH y presenta los resultados con claridad.

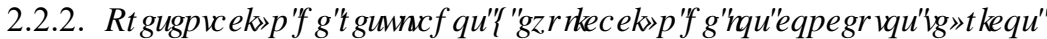

Los estudiantes que participaron como sujetos experimentales en el juego-experimento fueron tomando unas decisiones en cada situación financiera planteada, y viendo sus consecuencias. Esas decisiones y sus resultados se presentaron a la clase de forma agregada y se analizaron. Esto permitió observar las pérdidas y las ganancias que habían ocasionado dichas decisiones. En ese punto, los estudiantes estaban muy interesados en saber el por qué y, por tanto, se mostraron muy receptivos a las explicaciones de los conceptos teóricos más importantes relacionados con el área de estudio. Al finalizar la exposición, la docente y el alumnado intercambiaron impresiones sobre el área de estudio, añadiendo ejemplos de la realidad, y favoreciendo un debate ágil y dinámico en torno a los asuntos más destacados.

\subsection{Redacción del informe}

Finalizadas las exposiciones, los alumnos presentaron en el plazo de una semana un resumen ejecutivo que debía contener: en primer lugar, una explicación clara del experimento económico realizado, sus objetivos y resultados; y, en segundo lugar, una contextualización del área de estudio y los conceptos teóricos relacionados, aportando ejemplos de la vida real que permitiesen ilustrarlos. El contenido de los informes fue corregido por la docente, incorporando mejoras, y distribuido a todos los alumnos para su estudio, ya que formaba parte de la prueba final objetiva de la asignatura.

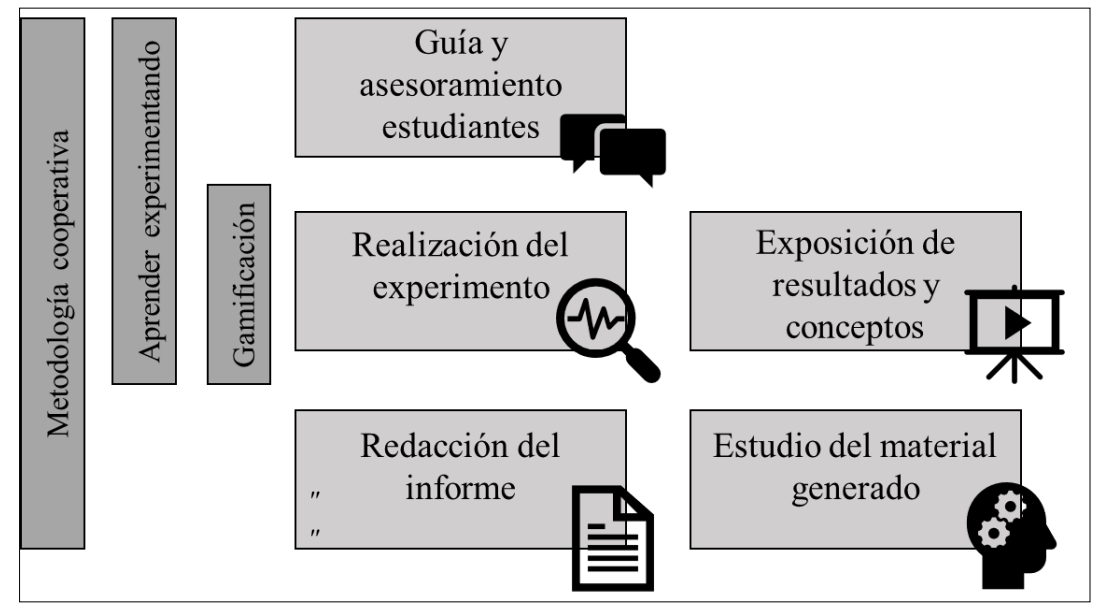

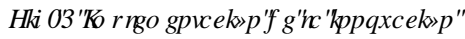

\section{Resultados}

La evaluación de los resultados de esta experiencia de innovación educativa se realizó por dos vías. Por una parte, se realizó un examen objetivo para medir los conocimientos y la comprensión adquirida sobre conceptos económico-financieros complejos, como el comportamiento de manada en los mercados RQDQH los comportamientos racionales e irracionales, las motivaciones que rigen la vida económica, y la 
formación de las burbujas financieras de precios, entre otros. Las excelentes respuestas obtenidas avalan la profunda comprensión que consiguieron los estudiantes.

Por otra parte, para medir la satisfacción e implicación de los estudiantes y sus percepciones sobre esta experiencia docente, se diseñó y pasó una encuesta anónima entre los estudiantes de la clase. Sus respuestas apuntan a niveles altos de satisfacción, no solo con los conocimientos adquiridos sino también con el dinamismo y carácter lúdico del proceso de aprendizaje. Como vemos en la Tabla 3, los estudiantes calificaron con un 4,7 sobre 5 el nivel de aprendizaje durante la realización del proyecto, con un 4,6 su rendimiento, y con un 4,7 la calidad del proyecto presentado en clase. Todo ello sugiere que el proceso de adquisición de conocimientos fue efectivo y, además, los estudiantes se esforzaron en la realización del proyecto, puesto que su impresión en cuanto al desempeño y al resultado fue ampliamente positiva.

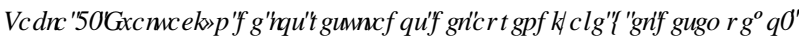

\begin{tabular}{lc}
\hline \multicolumn{1}{c}{ Pregunta } & $\begin{array}{c}\text { Puntuación } \\
\text { media }\end{array}$ \\
\hline Del 1 al 5, valora lo que has aprendido durante la realización del proyecto. & 4,7 \\
\hline Del 1 al 5, valora tu desempeño en la realización del proyecto. & 4,6 \\
\hline Del 1 al 5, valora la calidad del proyecto que has presentado en clase. & 4,7 \\
\hline
\end{tabular}

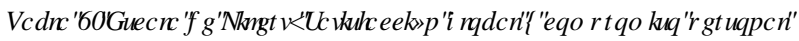

\begin{tabular}{|c|c|c|c|c|c|}
\hline Afirmación & $\begin{array}{l}\text { Muy de } \\
\text { acuerdo }\end{array}$ & $\begin{array}{c}\text { De } \\
\text { acuerdo }\end{array}$ & Neutral & $\begin{array}{c}\text { En } \\
\text { desacuerdo }\end{array}$ & $\begin{array}{c}\text { Muy en } \\
\text { desacuerdo }\end{array}$ \\
\hline $\begin{array}{l}\text { Considero que los experimentos son } \\
\text { útiles para interiorizar conceptos } \\
\text { económico-financieros. }\end{array}$ & $84,61 \%$ & $11,54 \%$ & $3,85 \%$ & - & - \\
\hline $\begin{array}{c}\text { En mi opinión, he dedicado el tiempo } \\
\text { necesario para la realización del } \\
\text { proyecto. }\end{array}$ & $57,69 \%$ & $30,77 \%$ & $3,85 \%$ & $7,69 \%$ & - \\
\hline $\begin{array}{l}\text { Podría definir con claridad los puntos } \\
\text { más importantes de cada proyecto } \\
\text { presentado en clase. }\end{array}$ & $15,38 \%$ & $65,38 \%$ & $19,24 \%$ & - & - \\
\hline $\begin{array}{l}\text { Valoro positivamente la metodología } \\
\text { usada en clase (experimentos } \\
\text { económicos). }\end{array}$ & $73,08 \%$ & $26,92 \%$ & - & - & - \\
\hline $\begin{array}{l}\text { Me gustaría que más asignaturas } \\
\text { incluyesen experimentos económicos } \\
\text { para explicar conceptos económico- } \\
\text { financieros. }\end{array}$ & $57,69 \%$ & $38,46 \%$ & - & $3,85 \%$ & - \\
\hline $\begin{array}{c}\text { Estoy satisfecho/a con el trabajo } \\
\text { realizado. }\end{array}$ & $61,54 \%$ & $38,46 \%$ & - & - & - \\
\hline $\begin{array}{l}\text { Mis compañeros han realizado } \\
\text { proyectos muy competentes. }\end{array}$ & $61,54 \%$ & $38,46 \%$ & - & - & - \\
\hline $\begin{array}{l}\text { El tiempo proporcionado para realizar } \\
\text { el trabajo ha sido adecuado. }\end{array}$ & $42,31 \%$ & $38,46 \%$ & $11,54 \%$ & $7,69 \%$ & - \\
\hline $\begin{array}{l}\text { Los recursos proporcionados (libros, } \\
\text { artículos, plataforma experimental } \\
\text { RQQQH han sido suficientes. }\end{array}$ & $42,31 \%$ & $42,31 \%$ & $15,38 \%$ & - & - \\
\hline
\end{tabular}


Por otro lado, la Tabla 4 recoge la valoración que los estudiantes otorgaron mediante una escala de Likert a distintas afirmaciones relacionadas con la satisfacción global y el compromiso personal. Para cada afirmación, los estudiantes debían indicar si estaban muy de acuerdo, de acuerdo, neutrales, en desacuerdo o muy en desacuerdo. En general, los estudiantes estuvieron muy de acuerdo con la utilidad de la metodología empleada, considerando que los experimentos habían sido útiles para interiorizar nociones económicas y financieras $(84,61 \%)$ y apreciando así su carácter cooperativo $(73,08 \%)$. La mayoría de los encuestados consideró que el tiempo proporcionado para realizar el trabajo había sido suficiente (el 42,31\% se mostró muy de acuerdo y 38,46\%, de acuerdo) así como los materiales facilitados (un $84,62 \%$ estuvo muy de acuerdo o de acuerdo con que los libros, artículos y demás materiales sugeridos habían sido adecuados).

En cuanto al compromiso personal, los estudiantes estuvieron satisfechos con el tiempo empleado en la realización del proyecto ( $88,46 \%$ muy de acuerdo y de acuerdo) así como satisfechos tanto con el trabajo en el que habían participado (61,54\% muy de acuerdo y $38,46 \%$ de acuerdo) como con los de sus compañeros (61,54\% muy de acuerdo y $38,46 \%$ de acuerdo). Los encuestados indicaron también que serían capaces de explicar los puntos más importantes de los proyectos presentados en clase $(65,38 \%$, de acuerdo). Por último, indicaron que les gustaría que otras asignaturas incluyesen esta metodología basada

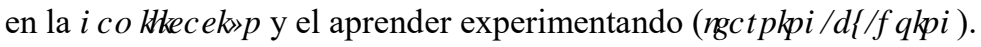

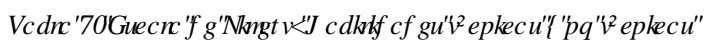

\begin{tabular}{|c|c|c|c|c|c|}
\hline Afirmación & $\begin{array}{l}\text { Muy de } \\
\text { acuerdo }\end{array}$ & $\begin{array}{c}\text { De } \\
\text { acuerdo }\end{array}$ & Neutral & $\begin{array}{c}\text { En } \\
\text { desacuerdo }\end{array}$ & $\begin{array}{c}\text { Muy en } \\
\text { desacuerdo }\end{array}$ \\
\hline $\begin{array}{l}\text { He mejorado mis habilidades } \\
\text { comunicativas. }\end{array}$ & $30,77 \%$ & $53,84 \%$ & $11,54 \%$ & $3,85 \%$ & - \\
\hline $\begin{array}{l}\text { He aprendido a responder con rapidez } \\
\text { ante situaciones imprevistas durante la } \\
\text { presentación de mi proyecto. }\end{array}$ & $30,77 \%$ & $46,15 \%$ & $23,08 \%$ & - & - \\
\hline $\begin{array}{l}\text { Después de la presentación del proyecto } \\
\text { he ganado seguridad en mí mismo/a. }\end{array}$ & $42,31 \%$ & $30,77 \%$ & $23,08 \%$ & $3,84 \%$ & - \\
\hline $\begin{array}{l}\text { Durante la presentación del proyecto, } \\
\text { me sentí inseguro/a. }\end{array}$ & $11,54 \%$ & $11,54 \%$ & $26,92 \%$ & $26,92 \%$ & $23,08 \%$ \\
\hline $\begin{array}{l}\text { Este proyecto me ha permitido trabajar } \\
\text { el pensamiento crítico. }\end{array}$ & $42,31 \%$ & $50,00 \%$ & $7,69 \%$ & - & - \\
\hline $\begin{array}{l}\text { Las habilidades desarrolladas me } \\
\text { ayudarán en mi futuro profesional. }\end{array}$ & $53,85 \%$ & $34,62 \%$ & $11,53 \%$ & - & - \\
\hline $\begin{array}{l}\text { Este proyecto me ha permitido salir de } \\
\text { mi zona de confort. }\end{array}$ & $38,46 \%$ & $53,85 \%$ & $7,69 \%$ & - & - \\
\hline
\end{tabular}

Por lo que respecta al desarrollo de habilidades, una gran mayoría de los encuestados (entre el $73 \%$ y el 92,31\%) estuvieron muy de acuerdo y de acuerdo con que habían mejorado sus habilidades comunicativas, aprendido a mejorar su capacidad para responder rápidamente a situaciones inesperadas, trabajado su capacidad crítica, abandonado su zona de confort, ganado confianza en ellos mismos y desarrollado habilidades que les ayudarían en su futuro profesional. Con respecto a la inseguridad experimentada durante la presentación, si bien el 50\% afirmó que estaba en desacuerdo o muy en desacuerdo con que se habían sentido inseguros durante la presentación, el 11,54\% indicó que estaba muy de acuerdo y otro $11,54 \%$ de acuerdo. 


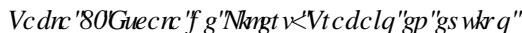

\begin{tabular}{|c|c|c|c|c|c|}
\hline Afirmación & $\begin{array}{l}\text { Muy de } \\
\text { acuerdo }\end{array}$ & $\begin{array}{c}\text { De } \\
\text { acuerdo } \\
\end{array}$ & Neutral & $\begin{array}{c}\text { En } \\
\text { desacuerdo } \\
\end{array}$ & $\begin{array}{c}\text { Muy en } \\
\text { desacuerdo }\end{array}$ \\
\hline $\begin{array}{l}\text { La dinámica de mi grupo de trabajo ha } \\
\text { sido satisfactoria. }\end{array}$ & $46,15 \%$ & $42,31 \%$ & $11,54 \%$ & - & - \\
\hline $\begin{array}{l}\text { Hemos realizado diversas reuniones en } \\
\text { las que hemos preparado el proyecto } \\
\text { trabajando en equipo. }\end{array}$ & $61,54 \%$ & $30,76 \%$ & $3,85 \%$ & $3,85 \%$ & - \\
\hline $\begin{array}{l}\text { En el equipo de trabajo, hemos sido } \\
\text { capaces de apoyarnos y motivarnos los } \\
\text { unos a los otros. }\end{array}$ & $57,69 \%$ & $26,92 \%$ & $11,54 \%$ & $3,85 \%$ & - \\
\hline $\begin{array}{l}\text { Hemos tenido dificultades a la hora de } \\
\text { encontrar fuentes fiables de las que } \\
\text { obtener información veraz. }\end{array}$ & $3,85 \%$ & $11,54 \%$ & $11,54 \%$ & $42,31 \%$ & $30,77 \%$ \\
\hline $\begin{array}{l}\text { Hemos dividido el proyecto en distintas } \\
\text { partes y cada miembro del grupo se ha } \\
\text { encargado exclusivamente de su parte. }\end{array}$ & $26,92 \%$ & $19,23 \%$ & $30,78 \%$ & $15,38 \%$ & $7,69 \%$ \\
\hline $\begin{array}{l}\text { He conocido mejor a mis compañeros } \\
\text { gracias a las presentaciones. }\end{array}$ & $65,39 \%$ & $26,92 \%$ & $7,69 \%$ & - & - \\
\hline $\begin{array}{c}\text { Durante el trabajo en equipo, un } \\
\text { miembro ha adoptado informalmente el } \\
\text { rol de líder. }\end{array}$ & $23,08 \%$ & $38,46 \%$ & $26,92 \%$ & $7,69 \%$ & $3,85 \%$ \\
\hline $\begin{array}{l}\text { Me gustaría haber escogido a mis } \\
\text { compañeros de equipo. }\end{array}$ & $23,08 \%$ & $11,54 \%$ & $26,92 \%$ & $23,08 \%$ & $15,38 \%$ \\
\hline
\end{tabular}

Por último, los estudiantes puntuaron una serie de afirmaciones sobre el trabajo en equipo (ver Tabla 6). Estuvieron muy de acuerdo con que las dinámicas de grupo habían sido satisfactorias y con que habían sido capaces de apoyarse y motivarse entre ellos. Además, estuvieron muy de acuerdo con que el proyecto les había permitido conocer mejor a sus compañeros. Preguntados por las dificultades a la hora de encontrar fuentes fiables y útiles de información, el 73,08\% estuvo muy en desacuerdo o en desacuerdo con haberlas experimentado, aunque un 15,39\% se mostró de acuerdo o muy de acuerdo, por lo que sería conveniente ofrecerles una guía más concreta sobre fuentes bibliográficas. No hubo consenso acerca de la división del trabajo, siendo la posición neutral la más representativa en la afirmación "dividimos el trabajo entre los miembros del grupo". Sin embargo, aquellos que optaron por dividir el trabajo, y por tanto estuvieron muy de acuerdo o de acuerdo con la afirmación, supusieron un $46,15 \%$. De este modo, la división del trabajo fue la alternativa más usada en detrimento de la realización del trabajo de forma conjunta. Por último, todo apunta a que afloraron roles de liderazgo, observándose posiciones encontradas sobre si les gustaría o no elegir a sus compañeros (el $34,62 \%$ estuvo de acuerdo o muy de acuerdo y el $38,46 \%$ en desacuerdo o muy en desacuerdo).

\section{Conclusiones}

La concesión de los premios Nobel de Economía a Vernon L. Smith (2002), Daniel Kahneman (2002), Robert H. Thaler (2017), Michael Kremer (2019), Abhijit Banerjee (2019) y Esther Duflo (2019) confirman que las decisiones económicas y financieras incorporan consideraciones sociales y psicológicas.

Para que los estudiantes conozcan y experimenten en sus propias decisiones las finanzas conductuales, y con ello entiendan mejor el comportamiento de los agentes económicos en distintas situaciones como subastas, fijación de precios, negociaciones, inversiones y financiación, hemos aplicado una innovación 
educativa que aúna tres metodologías: aprender experimentando, cooperar con el equipo, y aprender jugando en entornos digitales (JDP IIFDFly $Q$. Experimentando y jugando los alumnos consolidan firmemente conceptos económico-financieros de cierta complejidad debido a que descubren por ellos mismos sus implicaciones, entendiendo mejor y recordando por más tiempo los conocimientos adquiridos. Esta experiencia docente ha permitido comprobar las bondades de la JDP IIFDFIyQen entornos digitales para realizar experimentos económicos en clase de forma sencilla e intuitiva. Adicionalmente, los alumnos han desarrollado no solo habilidades técnicas y de conocimientos, sino también habilidades relacionadas con el liderazgo, el trabajo en equipo y la comunicación.

\section{Referencias}

AL-AZAWI, R., AL-FALITI, F. y AL-BLUSHI, M. (2016). "Educational Gamification Vs. Game Based Learning: Comparative Study” en ,QUAQDWRQDO-RXLQDORI, QQRYDURQDO DQDJ HP HQWDQGJ7HKQRRIJ, vol. 7, issue 4, p. 132136.

COHN, C.L. (1999). "Cooperative Learning in a Macroeconomics Course: A Team Simulation" en \&RØHH 7HFKLU, vol. 47, issue 2, p. 51-54.

COMEIG, I. y JARAMILLO-GUTIÉRREZ, A. (2011). "Experimentos interactivos para la enseñanza de economía y finanzas: una aplicación a la docencia de estadística" en Pavía, J.M., Martínez, R. y Morillas, F.G. ( [SHUHDFDWGH IQQRYDFIY QGRFHQMHHQHMDOWHFD. València: Universitat de València, p. 191-199.

GILLIES, R.B. (2016). "Cooperative Learning: Review of Research and Practice" en \$XWWDDQQ-RXLQDORID7HDFHU ( GXFDMRQ vol. 41, issue 3, p. 39-54.

HACKATHORN, J., SOLOMON, E. D. y BLANKMEYER, K. L. (2011). "Learning by Doing: An Empirical Study of Active Teaching Techniques" en 7KH-RXLQDORIC IIHFUHH7HDKLQJ, vol. 11, issue 2, p. 40-54

HOLT, C.A. (2005). 9HFRQDEIL( [SHUP HQWDO FRQRP IFWV DERWWRUTKveconlab.econ.virginia.edu>

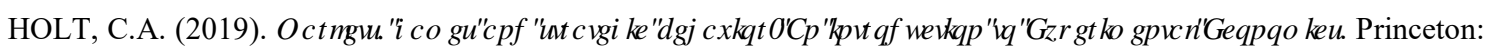
Princeton University Press.

RAMÍREZ, F. y GRAU, A. J. (2016). "La Innovación Educativa y la Economía Experimental: Un experimento sobre el contagio del pánico bancario” en Díaz-Cuesta Galián, J., Del Valle Mejías, M.E. y Linares Herrera, M. ,QQRYDFIyQ

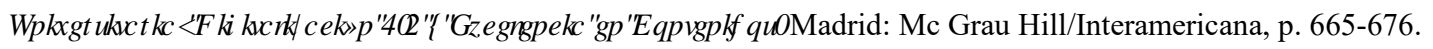

SMITH, V. (1994). "Economics in the Laboratory" en -RXLQDORIL FRQRP IFI3 HUSHFWHH, vol. 8, issue 1, p. 113-131. 